\title{
Electrostatic shock properties inferred from AKR fine structure
}

\author{
R. Pottelette ${ }^{1}$, R. A. Treumann ${ }^{2}$, M. Berthomier ${ }^{3}$, and J. Jasperse ${ }^{4}$ \\ ${ }^{1}$ CETP-CNRS, 4 Rue de Neptune, F-94107 Saint-Maur des Fossés, France \\ ${ }^{2}$ Centre for Interdisciplinary Plasma Science, Max-Planck-Institute for extraterrestrial Physics, P.O.Box 1312, \\ D-85748 Garching, Germany. Also at International Space Science Institute, Hallerstr. 6, CH-3012 Bern, Switzerland \\ ${ }^{3}$ Space Sciences Laboratory, University of California, Berkeley, CA 94720-7450, USA. On leave from CETP \\ ${ }^{4}$ Air Force Research Laboratory, Hanscom Air Force Base, MA 01731-3010, USA
}

Received: 31 January 2002 - Revised: 5 April 2002 - Accepted: 12 April 2002

\begin{abstract}
The auroral kilometric radiation (AKR) consists of a large number of fast drifting elementary radiation events that have been interpreted as travelling electron holes resulting from the nonlinear evolution of electron-acoustic waves. The elementary radiation structures sometimes become reflected or trapped in slowly drifting larger structures where the parallel electric fields are located. These latter features have spectral frequency drifts which can be interpreted in terms of the propagation of shock-like disturbances along the auroral field line at velocities near the ion-acoustic speed. The amplitude, speed, and shock width of such localized ionacoustic shocks are determined here in the fluid approximation from the Sagdeev potential, assuming realistic plasma parameters. It is emphasized that the electrostatic potentials of such nonlinear structures contribute to auroral acceleration.
\end{abstract}

\section{Introduction}

The FAST spacecraft, with its high time-resolution instrumentation and high telemetry rate, has provided new details concerning the complex plasma processes involved in the wave-particle interaction processes in the center of the auroral acceleration region. This region is marked by the transition layer from the hot, tenuous magnetospheric plasma to the cold and dense ionospheric plasma. In this paper, we concentrate on the study of the physical processes in the upwardcurrent region, the region that is associated with the visible aurora. It contains the convergent electrostatic fields (sometimes referred to as electrostatic shocks) and parallel potential drops. The FAST observations have demonstrated unambiguously that these parallel potential drops constitute the source of auroral potential acceleration (Carlson et al., 1998). They accelerate the precipitating magnetospheric electrons downward towards the ionosphere, where they cause the au-

Correspondence to: R. Pottelette (pottelet@ipsl.cetp.fr) rora and, vice versa, they accelerate the cold ionospheric ion population into an energetic, though cool upward ion beam.

Use of a single satellite, however, inhibits inferring about the dynamics and spatial scales of the parallel electric potential layers. In order to have access to such a piece of information, an alternative way consists of monitoring the time variation of the frequency drift of the fine structure detected in the spectrum of the auroral kilometric radiation (AKR). As previously shown, the analysis of these fine structures allows one to obtain information on the auroral acceleration processes, and to qualitatively infer about the spatial extension and the dynamics of the mesoscale field-aligned potential drops (Gurnett and Anderson, 1981; Pottelette et al., 2001).

The AKR, a most powerful radio emission of about a kilometric in wavelength, is one of the most spectacular phenomena of the auroral zone. Occasionally, the FAST spacecraft has crossed the AKR source region. The FAST observations confirmed many of the fundamental elements of the electron-cyclotron maser mechanism, usually acknowledged as the generation mechanism, but with substantial modification (Pritchett, 1984; Strangeway, 1985; Pritchett et al., 1999; Ergun et al., 2000). The most important of these modifications is that the emissions do not draw their energy from the loss-cone distribution of the radiating electrons. Rather, the radiation results from an unstable "horseshoe" distribution. Such a distribution is generated in the presence of an upward directed parallel electric field that accelerates plasma sheet electrons downward to form a beam-like distribution. When these electrons move down the field lines into increasing magnetic field strength, conservation of their first adiabatic invariant causes their pitch angles to increase as a consequence of the "mirror force". In these circumstances, the energetic electron distribution develops a peak at large pitch angles. This produces an extended region on the distribution function with $\partial F\left(v_{\|}, v_{\perp}\right) / \partial v_{\perp}>0$, which is the condition for the generation of radio waves. The weakly relativistic theory (Pritchett, 1984) shows that the radio-wave emission is very close to the direction perpendicular to the magnetic field. Moreover, the emission is in the X-mode and, in the 

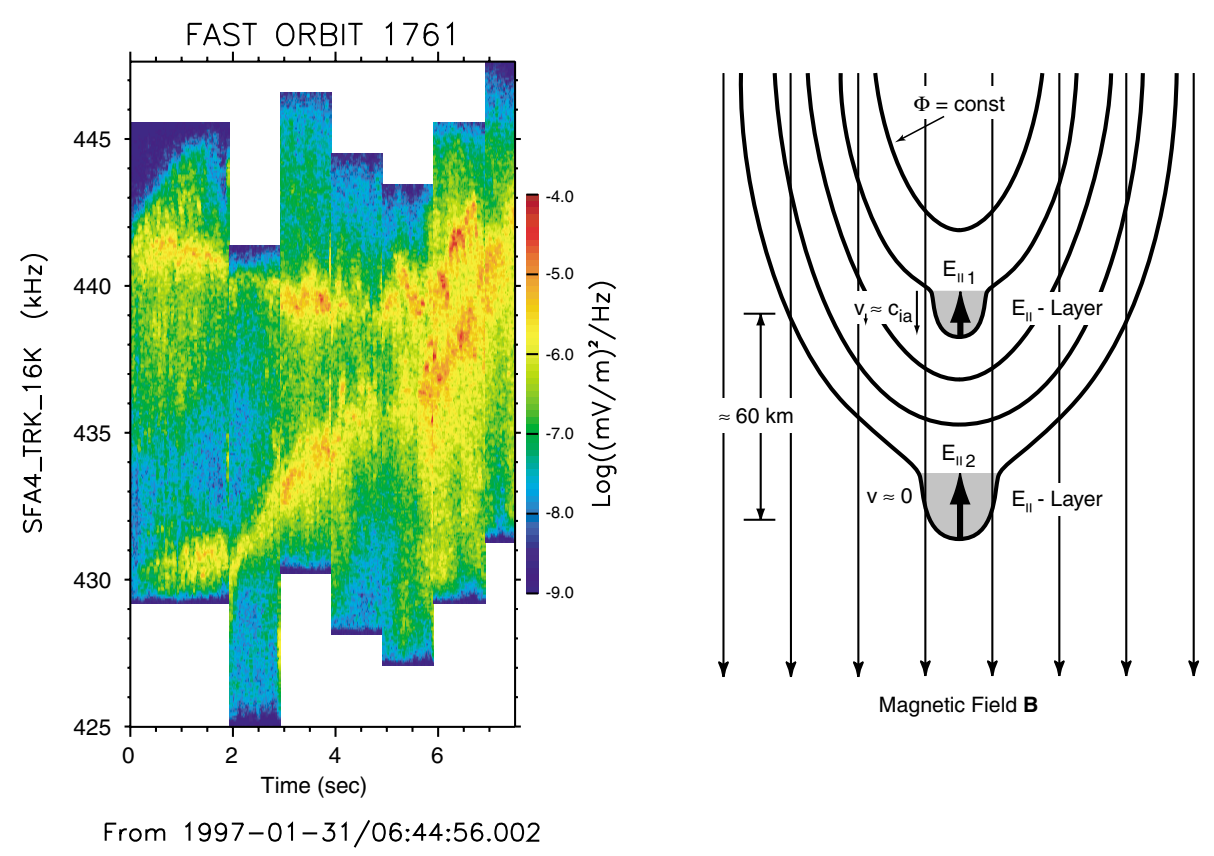

Fig. 1. Left: High time and frequency resolution recordings of AKR as obtained on 31 January 1997 by the FAST satellite above the auroral zone. Two narrow-band AKR emissions are visible at $430 \mathrm{kHz}$ and $440 \mathrm{kHz}$, approaching each other within $\sim 5 \mathrm{~s}$. As these emissions are generated at the local electron gyrofrequency, the upper band corresponds to a source spatially located below the one of the lower band. The upper band is nearly stable until after $6 \mathrm{~s}$, when the lower band approaches it and pushes it to higher frequencies. The two bands do not merge into one single band, suggesting that their polarities are repulsive. Right: A simple visualization of the presumable electric field structure where two field-aligned potential layers are sitting on one field line.

source region, has escaping frequency below the local nonrelativistic cyclotron frequency. The most far reaching implication (Ergun et al., 2000) is that the electron-cyclotron maser mechanism is directly associated with charged particle acceleration in magnetic field-aligned potential drops. The AKR is generated in those regions where the parallel electric fields are located. Being a direct consequence of the parallel acceleration processes, the radiation contains fundamental information on their characteristic spatial and temporal scales. In the remainder of this paper, we investigate these scales using the AKR fine structure observations of FAST as a tool for remote sensing of the acceleration region.

\section{Observations}

The AKR does not represent a continuous broad band emission (Gurnett and Anderson, 1981). Instead, early high timeresolution wide band measurements by the ISEE 1 and 2 spacecraft, performed at large distance from the AKR source region, showed that the radiation consists of many discrete narrow band emissions. The center frequency of the individual emissions often varies in a systematic manner, sweeping either upward or downward across the spectrum. The FAST observations allowed for a detailed study of the complex spectral structure of AKR. Pottelette et al. (2001) showed that the AKR emissions consist of a large number of fast drifting (what they called) "Elementary Radiation Events". They interpreted these EREs as travelling electron holes. The
ERE structures are sometimes reflected or trapped in slower drifting larger scale structures. These latter structures have been interpreted as parallel electric field layers. Figure 1 shows an example of such narrow-band slowly-drifting features detected by the on-board wave-tracker instrumentation on FAST. The bandwidth of each tracker snapshot is $16 \mathrm{kHz}$, with $100 \mathrm{~Hz}$ resolution, and the time resolution-per-sweep amounts to $16 \mathrm{~ms}$.

The tracker data on the left of Fig. 1 show two simultaneous narrow-band slowly-drifting emission features at different central frequencies. According to the theory of AKR generation (see e.g. Pritchett, 1984; Strangeway, 1985; Ergun et al., 2000), these two emissions are excited at, or just beneath, the local electron gyrofrequency $\omega_{c e}$ in regions containing field-aligned electric potential drops. The bandwidths of the emissions can be roughly identified with the variation of the local gyrofrequency along the radial extension of the parallel electric field layer. The two drifting features in Fig. 1 are highly variable in bandwidth, with the largest value being about $\sim 1 \mathrm{kHz}$. At the altitudes probed by FAST, this bandwidth corresponds to an extension of a few $\mathrm{km}$ along the magnetic field. The right part of Fig. 1 gives a schematic view of the dynamics and interaction of two such emission layers (Pottelette et al., 2001). When FAST first detected the event, the two layers in Fig. 1 were separated by a frequency gap of $\sim 12 \mathrm{kHz}$, corresponding to a mutual distance of about $60 \mathrm{~km}$ along the magnetic field line. The high-frequency feature, which is believed to be located at lower altitude, initially 


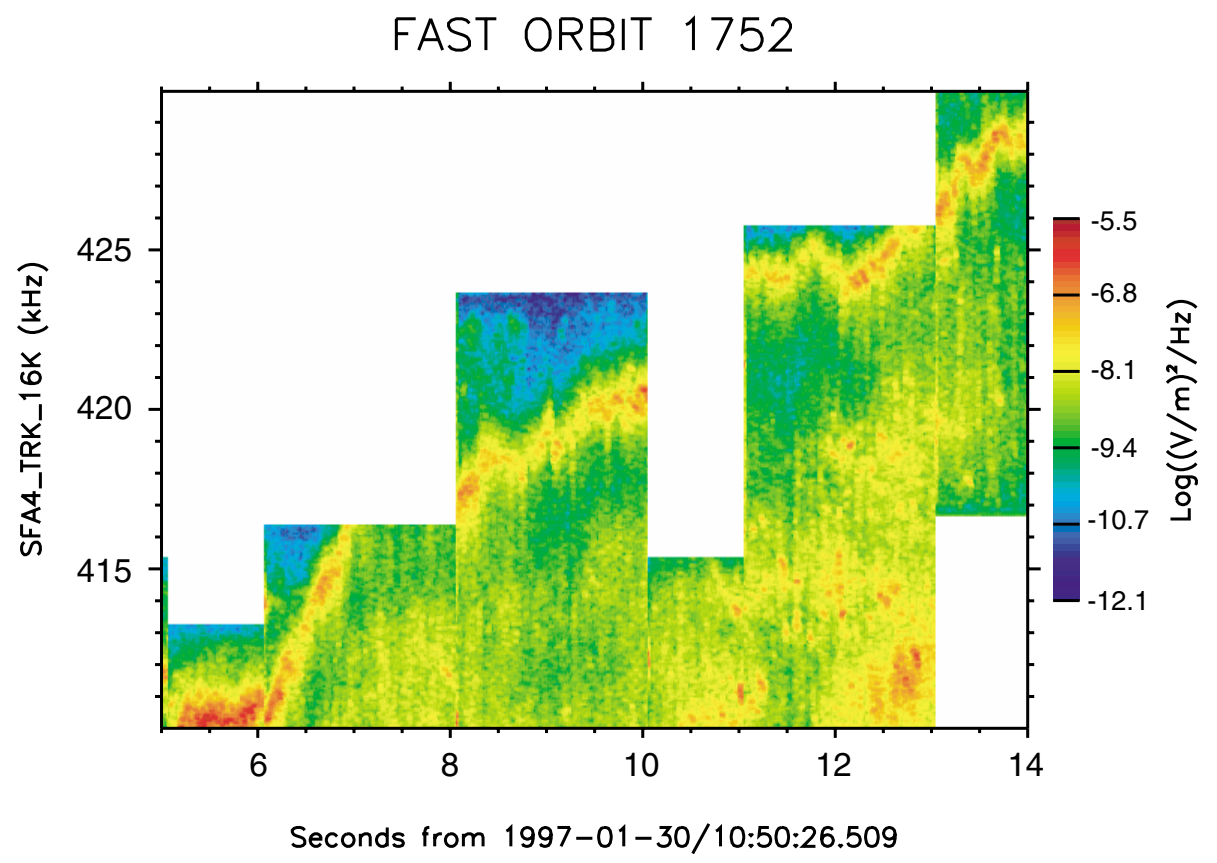

Fig. 2. Spectral dynamics of a narrow emission band in auroral kilometric radiation. Though the emission band remains stable over the entire period of $9 \mathrm{~s}$, its drift across the spectrum experiences severe changes in time. In the average the band is moving towards lower altitudes (increasing frequency) suggesting that the source is riding on the auroral electron beam in the upward current AKR source region. The average drift speed re-calculated into real space corresponds to the ion-acoustic velocity.

moved slowly upward in real space, as indicated by the decrease in its central emission frequency. The rate of decrease is of the order of $\sim 1 \mathrm{kHz} \mathrm{s}^{-1}$, corresponding to a velocity of $\sim 10 \mathrm{~km} \mathrm{~s}^{-1}$ along the field. At the same time, the lowfrequency feature that is located at the higher altitude moved downward in real space, as indicated by the increasing central spectral emission frequency. Its spectral frequency drift is about constant during this period and corresponds to the somewhat larger velocity of about $\sim 40 \mathrm{~km} \mathrm{~s}^{-1}$. At the end of the event, when the two features approach each other, they start moving together, though remaining mutually separated at a constant separation distance of $\sim 3 \mathrm{kHz}$, corresponding to roughly a $15 \mathrm{~km}$ vertical distance. It is important to note that the two radiation sources do not merge. Instead, the lowfrequency emission region that is approaching the higher frequency source from above obviously pushes the latter down the field line. These observations can be interpreted as the interaction of two parallel electric field layers of the same polarity, with the upper source region having a larger momentum than the lower region.

Figure 2 shows another example related to the dynamics of such localized electric field structures. For the $9 \mathrm{~s}$ duration where the wave tracker could follow this event, the structure is moving predominantly downward at a velocity ranging from $\sim 10-80 \mathrm{~km} \mathrm{~s}^{-1}$ with the higher speed attained at larger altitudes. The displacement speed is highly variable in the present case, which indicates a considerable dynamics in the source region. The range of the velocities observed inhibits an easy identification of the emission-source struc- ture with a particular wave mode excited in a Maxwellian plasma as a causative agent. However, it roughly matches the range of ion-acoustic waves for the parameters of the lower magnetospheric source region of AKR. Therefore, tentatively suggest that the slowly-drifting emission bands in the AKR may consist of nonlinear ion-acoustic features evolving under the local conditions that are met in the auroral plasma. The most probable of those features are ion-acoustic solitons (e.g. Buti, 1980; Reddy and Lakhina, 1991; Reddy et al., 1992) which may evolve into ion holes (e.g. Gray et al., 1991). The latter form during the nonlinear evolution of ionacoustic waves in the presence of field-aligned currents and are a typical consequence of the nonlinear interaction of ionacoustic waves with a diluted hot plasma.

Field-aligned currents have been proposed long ago by Kindel and Kennel (1971) as the free energy source of the ion-acoustic instability. Subsequent two-dimensional numerical simulations (e.g. Gray et al., 1991) indeed showed that current-generated ion-acoustic waves of high current drift speed $v_{\text {FAC }}>c_{i a}$, well above the ion-acoustic threshold, readily evolve into ion holes. Numerical simulations by Berman et al. (1985) suggested that the originally high threshold for instability is substantially lowered by the development of holes. This would rule out the conclusion of most authors that ion-acoustic waves merely play a secondary role in auroral dynamics, thereby giving the most attention to electrostatic ion-cyclotron waves (EICW). Indeed, relatively large-amplitude EICW have been observed in the auroral plasma. However, these waves are mostly harmonic 
and have little tendency to form nonlinear structures, such as solitons. Since their electric fields are about perpendicular to the magnetic field, their effect on the electron component and, thus, on electron acceleration, should be small. It is probably reasonable to assume that they are not responsible for the presence of localized electric potential drops. On the other hand, ion-hole formation makes the threshold of ion-acoustic waves practically vanish, allowing for short parallel-wavelength ion-acoustic waves to be excited locally already at a very weak field-aligned current intensity. These appear as spatially localized electric fields, i.e. as ion holes that form on the cool plasma ion background: in our case, on the cool upward ion beam plasma. The holes survive for an extended time while moving into the warm electron background: in our case, the auroral horseshoe distribution. Lacking positively charged ions, the holes form a localized negative potential bump and are thus retarded by the current electrons. Interacting holes experience strong variations in their drift velocities. They even may reverse direction while maintaining their physical integrity. This is possible when the velocity change happens on a time scale faster than the decay time of the hole.

It must, however, be noted that Berman et al. (1985) were using an unrealistically low mass ratio of 4 (pointed out to us by the referee). Simulations with larger mass-ratios did indeed confirm the lowering of the threshold (Hudson et al., 1983) by the presence of holes. However, the initial evolution of the ion-acoustic waves still requires large initial current drifts, in which case, according to linear theory, the EICW should dominate. In the case under consideration here, the downward current drift velocity is of the order of $10000 \mathrm{~km} \mathrm{~s}^{-1}$, large enough, though still below the thermal electron speed, to provide a susceptible growth to ion-acoustic waves, and probably fast enough to overcome the growth of EICW. These waves should, after a few ion plasma periods, readily enter the nonlinear regime, when they will non-resonantly evolve into solitons and ion holes, thereby further lowering the instability threshold. This evolution can be handled in terms of a two-component fluid theory (Berthomier et al., 1998). In the following section, we use this theory in order to investigate the properties of a well developed auroral electrostatic shock.

\section{Inferred electrostatic shock properties}

Large-amplitude density depletions of the ion-acoustic type can be excited in plasmas exhibiting two Maxwellianelectron populations, provided that the temperature ratio of the two electron populations is sufficiently high (Buti, 1980). The auroral plasma consists of a mixture of a minor cold $(\sim \mathrm{eV})$ electron component and of a predominant hot $(\sim \mathrm{keV})$ electron component. It can thus be expected that such type of nonlinear structures are likely to develop in the auroral plasma. Berthomier et al. (1998) have shown that for large velocity, the nonlinear ion-acoustic hole structures evolve into electric potential ramps, which are typical for so-called

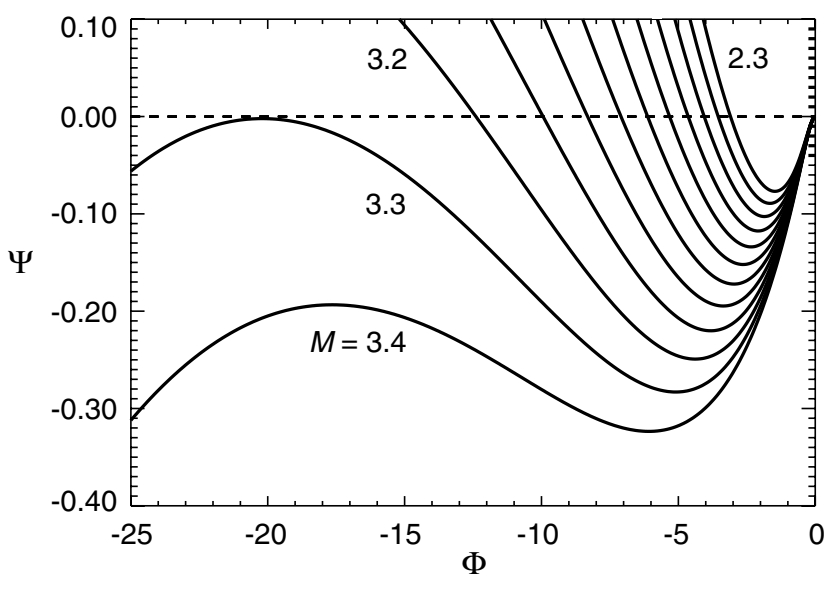

Fig. 3. The pseudo-potential $\Psi(\Phi)$ as function of the normalized electrostatic potential $\Phi(\phi)$. The calculation has been performed for $n=n_{h} / n_{c}=5, t \equiv t_{h} / t_{c}=200$, and $t_{i}=0.1$. Solutions are only for negative values $\Psi<0$ of $\Psi$. Finite "discrete" (or "harmonic") solutions are obtained in the range of Mach numbers corresponding to the localized minima in $\Psi$. Those solutions are supersonic and correspond to solitary structures. Electrostatic shock solutions are possible close to the upper limit of the allowed Mach number, which, in this case, is $M_{S} \approx 3.3$. Close to $M_{s}$ the potential becomes asymmetric, and a real field-aligned potential drop builds up across the structure.

electrostatic shocks with finite net potential drop across the structure. Such drops are needed for the acceleration of electrons and ions, and may just constitute the observed fieldaligned drifting potential features. In the following, based on the above theoretical approach and observations, we estimate the order of magnitude of the amplitude of such electrostatic shocks for the particular case of our observations.

Let us consider a collisionless plasma consisting of a single cool ion component and two thermal electron populations. The cold electron population, which has a temperature of a few $\mathrm{eV}$, typical for the ionospheric electron population, is assumed to be highly dilute, while the predominant hot $\sim$ keV-temperature electron component represents the precipitating plasma-sheet horseshoe electrons. Each electron population is assumed to be in equilibrium in the electrostatic potential $\phi$. The electron densities of the cold and hot components, $n_{c}$ and $n_{h}$, obey the Boltzmann relation, with the respective temperatures $T_{c}$ and $T_{h}$.

We define the normalized potential $\Phi=e \phi / k_{\mathrm{B}} T_{\mathrm{eff}}$, the normalized electron temperatures $t_{c h}=T_{c h} / T_{\text {eff }}$, where $T_{\text {eff }}=T_{c} T_{h} /\left[\left(n_{c} / n_{0}\right) T_{h}+\left(n_{h} / n_{0}\right) T_{c}\right]$ is the efficient temperature corresponding to the ion-acoustic speed $c_{\text {eff }}$, excited in such a plasma, and $n_{0}=n_{c}+n_{h}$ is the total electron density. The normalized fluid equations can be reduced to

$$
\begin{aligned}
& \frac{\partial n_{i}}{\partial t}+\frac{\partial\left(n_{i} v_{i}\right)}{\partial x}=0 \\
& \frac{\partial v_{i}}{\partial t}+v_{i} \frac{\partial v_{i}}{\partial x}=-\frac{\partial \Phi}{\partial x}-3 t_{i} n_{i} \frac{\partial n_{i}}{\partial x}
\end{aligned}
$$




$$
\frac{\partial^{2} \Phi}{\partial x^{2}}=n_{c}+n_{h}-n_{i},
$$

where we normalized the densities to $n_{0}$, lengths to the effective Debye length $\lambda_{\mathrm{D} \text {,eff }}$, velocities to the effective ionacoustic speed $c_{\text {eff }}=\sqrt{k_{\mathrm{B}} T_{\text {eff }} / m_{i}}$, and temperatures to $T_{\text {eff }}$. The time is taken in units of the inverse ion plasma frequency $\omega_{p i}$. Furthermore, we assume that the ion population responds adiabatically. We are looking for waves travelling along the magnetic field direction $x$, and transforming to the moving system $x \rightarrow x-M t$, where $M=V_{0} / c_{\text {eff }}$ is the Mach number of the stationary wave structure, and $V_{0}$ its velocity. In the stationary case, the one-dimensional set of the above equations is reduced to

$\frac{1}{2}\left(\frac{\mathrm{d} \Phi}{\mathrm{d} x}\right)^{2}+\Psi(\Phi)=0$.

This equation is similar to that of an imaginary particle with position $\Phi$ and velocity $\mathrm{d} \Phi / \mathrm{d} x$ moving in the so-called Sagdeev pseudo-potential $\Psi$ and has been solved analytically (Berthomier et al., 1998). The pseudo-potential $\Psi$ is given by

$$
\begin{array}{r}
\Psi(\Phi)=t_{c} \frac{n_{c 0}}{n_{0}}\left[1-\exp \frac{\Phi}{t_{c}}\right]+t_{h} \frac{n_{h 0}}{n_{0}}\left[1-\Phi \frac{\Phi}{t_{h}}\right] \\
-\left(3 t_{i} M^{6}\right)^{\frac{1}{4}}\left[\sqrt{\theta}-\sqrt{\theta_{0}}-\frac{1}{3}\left(\sqrt{\theta^{3}}-\sqrt{\theta_{0}^{3}}\right)\right]
\end{array}
$$

and $\theta$ is defined by

$\ln \theta=\cosh ^{-1}\left[\frac{M^{2}+3 t_{i}-2 \Phi}{\sqrt{12 t_{i}} M}\right]$

with $\theta_{0}=\theta(\Phi=0)$.

The result of the numerical solution of the above equation is illustrated in Fig. 3 for the parameter set $n=n_{h} / n_{c}=5$, $t \equiv t_{h} / t_{c}=200$, and $t_{i}=0.1$, where only negative values of $\Psi$ have to be considered. Figure 3 shows that the amplitude $\Phi$ of ion-acoustic solitary structures is an increasing function of the Mach number $M$. Sufficiently far below the critical "shock" Mach number $M_{s} \approx 3.3$, electrically neutral solitary structures are excited, lacking a net potential drop across the soliton, $\Delta \phi=0$, even though the solitons are very different in structure from the ordinary Korteweg-deVries or nonlinear Schrödinger-like solitons. The symmetry of the solitons below $M=M_{s}$ proves that there exist no electrostatic shock solutions.

Electrostatic shock structures with $\Delta \phi \neq 0$ occur for Mach numbers close to $M_{s}=3.3$ and $\Phi \sim-20$, when the pseudopotential $\Psi$-curve becomes tangent to the abscissa axis. The selected threshold value for shocks corresponds to $k_{\mathrm{B}} T_{c}=$ $5 \mathrm{eV}, k_{\mathrm{B}} T_{h}=1 \mathrm{keV}$, and an effective temperature of $k_{\mathrm{B}} T_{\mathrm{eff}}=$ $30 \mathrm{eV}$, which gives an ion-acoustic speed $c_{\text {eff }}=50 \mathrm{~km} \mathrm{~s}^{-1}$ and a density ratio $n=n_{h} / n_{0}=85 \%$. These are values that are typically met at the base level of the acceleration regions in the auroral magnetosphere (Pottelette et al., 1999).

Shock structures generated for $\Phi=-20$, granted that $k_{\mathrm{B}} T_{\text {eff }}=30 \mathrm{eV}$, correspond to a moderate electric-potential drop of $\phi \approx 600 \mathrm{~V}$. This estimate of half a $\mathrm{kV}$ potential drop across the electrostatic shock gives a relatively large value for the elementary potential drop supported by one such localized shock structure. This potential drop is, however, still too small for generating the observed parallel acceleration of auroral electrons, which, in many cases, may reach values of $10 \mathrm{keV}$. The observations do, however, show that, in most cases, not one single electric field layer evolves in the auroral magnetosphere. Instead, several such layers can be expected to be the rule. Evidence for their existence can be drawn from the observation of several simultaneous emission bands in the AKR at different central frequencies, as is found, for instance, from the observations in Fig. 1. The potentials of these layers along a magnetic field will add up to produce the energetic electrons in the energy range observed in the auroral magnetosphere.

One may then conclude that energetic electrons are produced by electric potential drops resulting from the spatial arrangement along one auroral flux tube of several microscopic electrostatic shock structures of the kind mentioned in this paper, when their potential drops add up along the field line over meso-scale distances. In order to estimate the number of such electrostatic shocks required to explain the observed acceleration of $\Delta E \sim$ few $\mathrm{keV}$, we need to estimate the spatial width $w_{s}$ of one of the electrostatic shocks. This can be done with the help of Eq. (4), which for an estimate can be rewritten for $w_{s}$ as

$w_{s} \approx|\Delta \Phi| /(2\langle|\Psi(\Phi)|\rangle)^{\frac{1}{2}}$,

holding for the curve corresponding to the shock Mach number $M_{S}$. The angular brackets indicate averaging over $\Phi$. From Fig. 3, we have $\Delta \Phi \approx-20,\langle\Psi\rangle \approx-0.15$. These values yield roughly $w_{s} \approx 50 \lambda_{\mathrm{D} \text {,eff }}$ for the width of the shock transition. For the conditions of Fig. 2, the input values are $f_{p e}=10 \mathrm{kHz}$ for the plasma frequency, $k_{\mathrm{B}} T_{\mathrm{eff}}=30 \mathrm{eV}$ for the temperature, and thus $\lambda_{\mathrm{D} \text {,eff }} \approx 35 \mathrm{~m}$, yielding a shock width of $w_{s} \approx 2 \mathrm{~km}$, and an electric field of $\sim 300 \mathrm{mV} \mathrm{m}^{-1}$, in excellent agreement with the observational conclusion from Fig. 2 that the shock thickness should be at most a few km wide. In our previous paper (Pottelette et al., 2001), we estimated the distance between any two such electrostatic shocks along the magnetic field to amount to some $\sim 10-100 \mathrm{~km}$.

It seems that a limited number of such electrostatic layers will evolve along a typical auroral flux tube. The number may be larger when the flux tube is wide enough. The typical total transverse width of such a flux tube containing the auroral acceleration region, as seen by FAST, is of the order of tens of seconds up to $1 \mathrm{~min}$, corresponding to transverse distances of up to a few $100 \mathrm{~km}$ crossed by the spacecraft at FAST altitudes. The lateral extension of any meso-scale electrostatic shock or electric field layer is much narrower. If we assume that it is a few times its field-aligned extension of the electric field layer, then it will be of several $\mathrm{km}$ across the magnetic field. Clearly, even if there are many such electric field layers in the total auroral acceleration region, their narrow lateral extension makes it plausible that in one of those auroral flux tubes, only a few shock layers 
will contribute to the potential ladder along the flux tube. If each of them contributes half a $\mathrm{kV}$ potential drop, this will readily explain that typical acceleration energies fall into the interval between several $100 \mathrm{eV}$ and several $\mathrm{keV}$. Therefore, it is not difficult to generate a total field-aligned potential drop of several $\mathrm{keV}$ as required by the observations of the particle acceleration, by hosting a small number of meso-scale electrostatic shocks along an auroral magnetic flux tube over a distance of a few $1000 \mathrm{~km}$.

\section{Conclusions}

Within the fluid approach of our model calculation, we have estimated the meso-scale electrostatic-shock amplitude that can be reached in the nonlinear evolution of an ion-acoustic wave that is excited in the two-electron component auroral plasma with the minor (ionospheric) cold (few eV) component and the dominant hot (few keV) component. The velocity of these structures is several times $c_{e f f}$ and depends sensitively on the respective density and temperature of the two electron populations. This dependence may explain the high variability of the speed of such structures, as derived from the measurement of the spectral frequency drifts observed in the AKR fine structure. Such a type of nonlinear structure is likely to develop on a field-aligned scale of several tens of effective Debye lengths.

The estimated amplitude of the meso-scale electrostatic shock associated with one localized nonlinear structure may be moderately large (corresponding to a few $100 \mathrm{eV}$ ). In order to accelerate auroral electrons up to the observed energies of $\sim 10 \mathrm{keV}$ a limited number of such electrostatic potentialdrop or electric-field layers located along one single auroral flux tube would be required.

Acknowledgement. We thank the referees for their constructive comments and improving suggestions. The FAST mission is a project of the Space Sciences Laboratory of the University of California at Berkeley run under the auspices of NASA. The authors are indebted to C. W. Carlson and R. E. Ergun for providing the particle and wave data as well as for some useful discussions. This research has been initiated within the France-Berkeley Program. Work of R.T. has been performed at ISSI, Bern. He thanks the directors of ISSI, J. Geiss, G. Paschmann, and R. von Steiger for their hospitality, and the ISSI staff for providing an excellent research atmosphere. Part of the work was supported by the PROCOPE cooperation under contract numbers D/9822921 and F/99057.

\section{References}

Berthomier, M., Pottelette, R., and Malingre, M.: Solitary waves and weak double layers in the two-electron-temperature auroral plasma, J. Geophys. Res., 103, 4261, 1998.
Berman, R. H., Tetreault, D. J., and Dupree, T. H.: Simulation of phase space density hole growth and the development of intermittent plasma turbulence, Phys. Fluids, 28, 155, 1985.

Buti, B.: Ion acoustic holes in a two temperature plasma, Phys. Lett. A, 76, 251, 1980.

Carlson, C. W., Pfaff, R. F., and Watzin, J. G.: The FAST Auroral SnapshoT (FAST) mission, Geophys. Res. Lett., 25, 2013, 1998.

Ergun, R. E., Carlson, C. W., McFadden, J. P., Mozer, F. S., Delory, G. T., Peria, W., Chaston, C. C., Temerin, M., Elphic, R., Strangeway, R., Pfaff, R., Cattell, C. A., Klumpar, D., Shelley, E., Peterson, W., Moebius, E., and Kistler, L.: FAST satellite observations of large amplitude solitary structures, Geophys. Res. Lett., 25, 2061, 1998.

Ergun, R. E., Carlson, C. W., McFadden, J. P., Delory, G. T., Strangeway, R. J., and Pritchett, P. L.: Electron-cyclotron maser driven by charged-particle acceleration from quasi-static magnetic-field-aligned potentials, Astrophys. J., 538, 456, 2000.

Gray, P. C., Hudson, M. K., Bergmann, R., and Roth, I.: Decay of ion beam driven acoustic waves into ion holes, Geophys. Res. Lett., 18, 1675, 1991.

Gurnett, D. A. and Anderson, R. R.: The kilometric radio emission spectrum: relationship to auroral acceleration processes, in Physics of auroral arc formation, Eds. Akasofu, S.-I. and Kan, J. R., AGU, Washington, D.C., Geophys. Monogr. 25, 341, 1981.

Hudson, M. K., Lotko, W., Roth, I., and Witt, E.: Solitary waves and double layers on auroral field lines, J. Geophys. Res., 88, 916, 1983.

Kindel, J. M. and Kennel, C. F.: Topside current instabilities, J. Geophys. Res., 76, 3055, 1971.

Pottelette, R., Ergun, R. E., Treumann, R. A., Berthomier, M., Carlson, C., McFadden, J. P., and Roth, I.: Modulated electron acoustic waves in auroral density cavities: FAST Observations, Geophys. Res. Lett., 26, 2629, 1999.

Pottelette, R., Treumann, R. A., and Berthomier, M.: Auroral Plasma Turbulence and the cause of AKR fine structure, J. Geophys. Res., 106, 8465, 2001.

Pritchett, P. L.: Relativistic dispersion, the cyclotron maser instability, and auroral kilometric radiation, J. Geophys. Res., 89, 8957, 1984.

Pritchett, P. L., Strangeway, R. J., Carlson, C. W., Ergun, R. E., McFadden, J. P., and Delory, G. T.: Free energy source and frequency bandwidth for the auroral kilometric radiation, J. Geophys. Res., 104, 10326, 1999.

Reddy, R. V. and Lakhina, G. S.: Ion acoustic double layers and solitons in auroral plasma, Planet. Space Sci., 39, 1343, 1991.

Reddy, R. V., Lakhina, G. S., and Verheest, F.: Ion-acoustic double layers and solitons in multispecies auroral beam-plasmas, Planet. Space Sci., 40, 1055, 1992.

Strangeway, R. J.: Wave dispersion and ray propagation in a weakly relativistic, nonequilibrium plasma: Implications for the generation of auroral kilometric radiation, J. Geophys. Res., 90, 9675, 1985.

Treumann, R. A. and Baumjohann, W.: Advanced Space Plasma Physics, Imperial College Press, London, 75-83, 261-268, 359361, 1997. 